Article

\title{
Optimal Patterned Wettability for Microchannel Flow Boiling Using the Lattice Boltzmann Method
}

\author{
Young Jin Wi, Jong Hyun Kim, Jung Shin Lee and Joon Sang Lee * \\ School of Mechanical Engineering, Yonsei University, Seoul 03722, Korea; yjwi17@naver.com (Y.J.W.); \\ whdgus@yonsei.ac.kr (J.H.K.); redljs@yonsei.ac.kr (J.S.L.) \\ * Correspondence: joonlee@yonsei.ac.kr; Tel.: +82-2-2123-5820
}

Received: 22 May 2018; Accepted: 5 August 2018; Published: 17 August 2018

\begin{abstract}
Microchannel flow boiling is a cooling method studied in microscale heat-cooling, which has become an important field of research with the development of high-density integrated circuits. The change in microchannel surface characteristics affects thermal fluid behavior, and existing studies have optimized heat transfer by changing surf ace wettability characteristics. However, a surface with heterogeneous wettability also has the potential to improve heat transfer. In this case, heat transfer would be optimized by applying the optimal heterogeneous wettability surface to channel flow boiling. In this study, a change in cooling efficiency was observed, by setting a hydrophobic and hydrophilic wettability pattern on the channel surface under the microchannel flow boiling condition, using a lattice Boltzmann method simulation. In the rectangular microchannel structure, the hydrophobic-hydrophilic patterned wettability was oriented perpendicular to the flow direction. The bubble nucleation and the heat transfer coefficient were observed in each case by varying the length of the pattern and the ratio of the hydrophobic-hydrophilic area. It was found that the minimum pattern length in which individual bubbles can occur, and the wettability pattern in which the bubble nucleation-departure cycle is maintained, are advantageous for increasing the efficiency of heat transfer in channel flow boiling.
\end{abstract}

Keywords: flow boiling; patterned wettability; microchannel flow; lattice Boltzmann method

\section{Introduction}

Microscale heat removal has played a critical role in determining the performance of high-density integrated circuits [1]. Furthermore, because there is limited steady air cooling, the cooling efficiency can be improved through the forced cooling of the artificial fluid flow in the cooling channel [2-4].

There has been extensive research to improve microchannel heat transfer efficiency. Most studies have focused on the modification of the surface structure, including the micro pin fin [5,6], cavity [7], and porous [8]. Deng et al. [5] used a laser micro-milling method to propose structured microchannels with micro cone pin fins. Xu et al. [6] studied the flow patterns during flow boiling instability in a microchannel with three types of pin fin arrays. Lin et al. [7] carried out an experimental study on convective boiling heat transfer and the CHF (Critical heat flux) of methanol-water mixtures in a diverging microchannel with artificial cavities. Deng et al. [8] investigated flow boiling heat transfer performance in a porous microchannel. As mentioned earlier, although micro structures in microchannels enhance heat transfer, it is challenging to fabricate a physical structure in a microchannel, and it takes considerable processing time to construct multiple channels.

Another method of research on surface characteristics that enhance boiling heat transfer is to use a surface coated with various materials. This modification can be easily achieved using a coating technique that does not involve any deformation of the surface [9] and can easily control the wettability. It is important that the characteristics of the two-phase flow are affected by the 
wettability of a surface. Zhou et al. [10] experimentally investigated the saturated flow boiling difference between the hydrophilic and super-hydrophilic surfaces in a rectangular microchannel. They concluded that the local dry out occurred on the hydrophilic surface at high heat fluxes for low mass fluxes and did not occur on the super-hydrophilic surface. Park et al. [11] also conducted an experimental study on the effect of flow boiling on wettability. They showed that the CHF is enhanced by a wettable surface. Li et al. [12] numerically investigated the boiling heat transfer for different levels of wettability and found that the CHF and wall superheat decreases at bad wettability. Gong et al. [13] conducted a numerical study on the effects of wettability during saturated pool boiling heat transfer. They demonstrated that a hydrophilic surface shows a higher onset of nucleate boiling temperature, lower heat transfer at low superheat, and higher CHF than a hydrophobic surface. Multiple studies on surfaces of homogeneous wettability have concluded that a hydrophilic surface has higher CHF and higher superheat than a hydrophobic surface [14,15]. The development of surface treatment technology enables the implementation of heterogeneous wettability surfaces $[16,17]$, and the pattern wettability has been studied as the method of overcoming the disadvantages of homogeneous wettability. Nam et al. [18] showed that the bubble nucleation speed on a surface with pattern wettability is higher than that on a surface with homogeneous wettability. Jo et al. [19] observed the changes in bubble formation and heat flux by controlling the size and arrangement of controlled hydrophobic patterns on a hydrophilic substrate. Lee et al. [20] employed a lattice Boltzmann method simulation to implement controlled hydrophobic patterns on a hydrophilic substrate and studied various modifications, such as to the shape and arrangement, to optimize pattern wettability.

In previous studies, patterned wettability has been found to influence bubble nucleation and the heat transfer coefficient during phase change. However, these studies focused on pool boiling, and it is necessary to also study the fluid flow in a channel. It is also important to establish a criterion for the optimized form in determining the pattern. In this paper, the effects of flow factors and patterns on the patterned wettability channel flow are investigated using a lattice Boltzmann method simulation, and the optimization of the patterned wettability in the channel flow is presented.

\section{Materials and Methods}

In this study, the modified pseudo-potential lattice Boltzmann model, which was proposed by Gong et al. [13,21], is used. This model includes a new interaction force using the exact difference method to minimize spurious velocity and increase the liquid-air density ratio. Other forcing schemes, such as that of Li et al. [22] or Xu et al. [23], can also be used to realize a large density ratio in a pseudo-potential model.

The derivation of the lattice Boltzmann equation through the discretization of the Boltzmann equation (the governing equation) can be found in the Appendix A [24], and the evolution equations for the flow and heat transfer are as follows:

$$
\begin{aligned}
& f_{i}\left(x+e_{i} \delta_{t}, t+\delta_{t}\right)-f_{i}(x, t)=-\frac{1}{\tau}\left[f_{i}(x, t)-f_{i}^{e q}(x, t)\right]+\Delta f_{i}(x, t) \\
& g_{i}\left(x+e_{i} \delta_{t}, t+\delta_{t}\right)-g_{i}(x, t)=-\frac{1}{\tau_{T}}\left[g_{i}(x, t)-g_{i}^{e q}(x, t)\right]+\delta_{t} \omega_{i} \phi
\end{aligned}
$$

Here, $f_{i}$ and $g_{i}$ are the density- and temperature-distribution functions, respectively. The evolution of these distribution functions depend upon the position, $x$, time, $t$, and relaxation times $\tau$ and $\tau_{T}$, where $\tau$ is the density-relaxation time and $\tau_{T}$ is the thermal relaxation time. The temperature fields of these media are solved concurrently because $\tau_{T}$ has different values for a working fluid and a solid substrate. The density- and temperature-equilibrium-distribution functions are:

$$
\begin{gathered}
f_{i}^{e q}=\omega_{i} \rho\left[1+3 e_{i} u+4.5\left(e_{i} u\right)^{2}-1.5 u^{2}\right] \\
g_{i}^{e q}=\omega_{i} T\left[1+3 e_{i} u_{\text {real }}+4.5\left(e_{i} u_{\text {real }}\right)^{2}-1.5 u_{\text {real }}^{2}\right]
\end{gathered}
$$


where $f_{i}^{e q}$ is the density-equilibrium distribution function and $g_{i}^{e q}$ is the temperature-equilibrium distribution function. For the three-dimensional lattice Boltzmann method using the D3Q19 model with distribution functions in 19 directions, the parameters $\omega_{\mathrm{i}}$ and $e_{i}$ are given as follows:

$$
\omega_{i}= \begin{cases}1 / 3, & i=0 \\ 1 / 18, & i=1, \cdots, 6 \\ 1 / 36, & i=7, \cdots, 18\end{cases}
$$

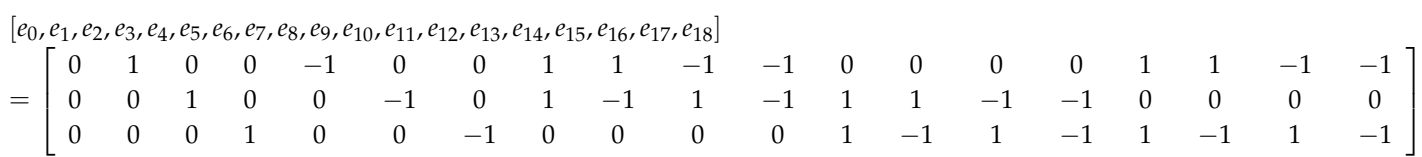

The body-force term is based on the exact difference method [25] and is calculated as follows:

$$
\Delta f_{i}(x, t)=f_{i}^{e q}(\rho(x, t), u+\Delta u)-f_{i}^{e q}(\rho(x, t), u)
$$

The velocity change, $\Delta u=F \cdot \delta t / \rho$, is calculated from the force $F$ :

$$
F=F_{\text {int }}(x)+F_{s}(x)+F_{g}(x)
$$

where $F_{\text {int }}$ is the interparticle-interaction force, given by:

$$
\begin{aligned}
F_{\text {int }}(x)= & -\beta \psi(x) \sum_{x^{\prime}} G\left(x, x^{\prime}\right) \psi\left(x^{\prime}\right)\left(x^{\prime}-x\right) \\
& -\frac{1-\beta}{2} \sum_{x^{\prime}} G\left(x, x^{\prime}\right) \psi^{2}\left(x^{\prime}\right)\left(x^{\prime}-x\right)
\end{aligned}
$$

Here, $\beta$ is based on Reference [26]. $G\left(x, x^{\prime}\right)$ has a nonzero value when $x$ and $x^{\prime}$ are adjacent to one another:

$$
G\left(x, x^{\prime}\right)= \begin{cases}g_{1}, & \left|x-x^{\prime}\right|=1 \\ g_{2}, & \left|x-x^{\prime}\right|=\sqrt{2} \\ 0, & \text { otherwise }\end{cases}
$$

where $g_{1}=g_{0}, g_{2}=g_{0} / 2$. for the D3Q19 scheme. The effective mass, $\psi(x)=\sqrt{2[p(x)-\rho(x)] /(6 g)}$, is based on the Peng-Robinson equation of state, as follows:

$$
p=\frac{\rho R T}{1-b \rho}-\frac{a \rho^{2} \phi_{0}(T)}{1+2 b \rho-b^{2} \rho^{2}}
$$

where $a=0.45724 R^{2} T_{c}^{2} / p_{c}, b=0.0778 R T_{c} / p_{c}$, and $\phi_{0}(T)$ is:

$$
\phi_{0}(T)=\left[1+\left(0.37464+1.54226 \omega-0.26992 \omega^{2}\right)\left(1-\sqrt{T / T_{c}}\right)\right]^{2}
$$

where $\omega=0.344$ is the acentric factor.

The wettability effect [27] is implemented in $F_{\mathrm{S}}$ and is given by

$$
F_{s}(x)=-\psi(x) \sum_{i} g_{s} \omega_{i} s\left(x+e_{i} \delta_{t}\right) e_{i} \delta_{t}
$$

The contact angles are controlled by the fluid-solid-interaction strength, $g_{s}$, and $s(x)$ is an indicator factor described as follows:

$$
s(x)= \begin{cases}0, & x \text { in fluid node } \\ 1, & x \text { in solid node }\end{cases}
$$

The gravitational force is given by: 


$$
F_{g}(x)=\left[\rho(x)-\rho_{\text {ave }}\right] g
$$

where $g$ is the gravitational acceleration and $\rho_{\text {ave }}$ is the average density.

The macroscopic equations for $u, T$, and $\rho$ are expressed as follows:

$$
\rho=\sum_{i} f_{i}, \quad \rho u=\sum_{i} e_{i} f_{i}, \quad T=\sum_{i} g_{i}
$$

The real fluid velocity, $u_{\text {real }}$, is obtained via force correction as follows:

$$
\rho u_{\text {real }}=\sum_{i} e_{i} f_{i}+\frac{\delta t}{2} F
$$

The thermal source related to phase change $\varphi$ is given by:

$$
\phi=T\left[1-\frac{1}{\rho c_{v}}\left(\frac{\partial p}{\partial T}\right)_{\rho}\right] \nabla \cdot u_{\text {real }}
$$

Figure 1 shows a schematic flow chart.

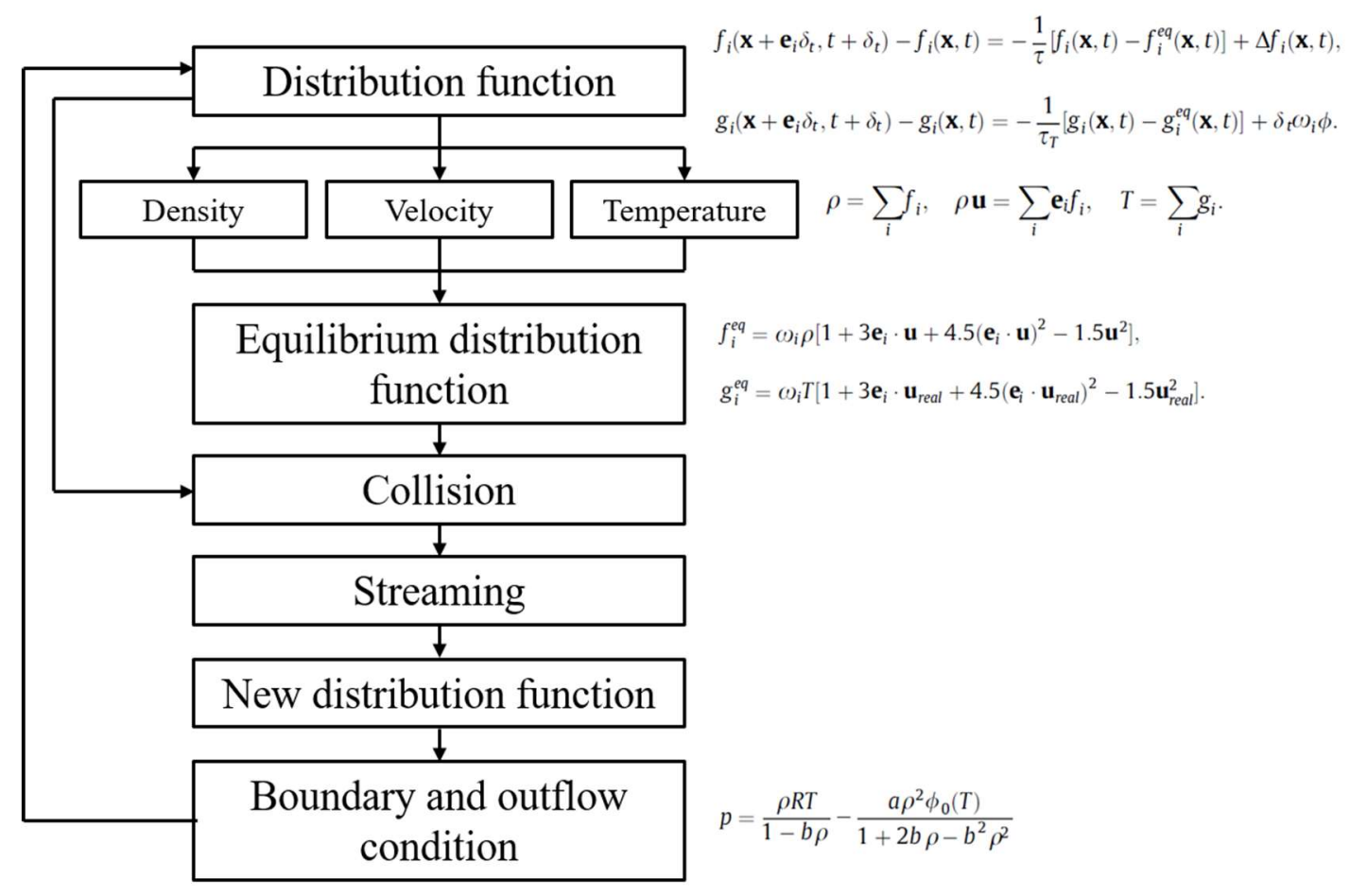

Figure 1. Lattice Boltzmann method flow chart.

\section{Simulation Domain Design}

In this numerical approach, the characteristic length $L_{0}$ is the capillary length:

$$
L_{0}=\sqrt{\frac{\gamma}{g\left(\rho_{1}-\rho_{g}\right)}}
$$

where $\gamma$ is the surface tension of the gas-liquid interface, and $\rho_{1}$ and $\rho_{\mathrm{g}}$ are the liquid and gas phase densities of water, respectively, at $100{ }^{\circ} \mathrm{C}$ and $1 \mathrm{~atm}$.

The normalized superheat, $T^{*}$, and the heat flux $Q^{\prime \prime}$ are: 


$$
T^{*}=\frac{\left(T-T_{\mathrm{s}}\right)}{T_{\mathrm{c}}}, Q^{\prime \prime}=\frac{Q L_{0}}{k T_{\mathrm{c}}}
$$

where $T_{\mathrm{s}}$ is the saturation temperature, $T_{\mathrm{c}}$ is the critical temperature, and $k$ is the thermal conductivity of liquid water at $100{ }^{\circ} \mathrm{C}$ and $1 \mathrm{~atm}[20]$.

Figure 2 shows the structure of the computational domain with a rectangular channel. Figure 2a shows the $x-y$ plane on the bottom channel surface $(z=0)$. The inlet, where fluid is applied to the channel, is located at $x=0$. The outlet where the channel flow escapes is located at $x=L_{l}$. There is a constant temperature boundary condition at $y=0$, and the walls spanning $y=L_{t}$ in the $x-y$ plane and spanning $y=L_{w}$ in the $y-z$ plane create a symmetric boundary condition. The boundary was applied to improve simulation efficiency, under the assumption that both sides of the channel shared the behavior. The hydrophobic-hydrophilic pattern is applied in a stripe formation on the bottom channel surface $(z=0)$. Other channel surfaces are hydrophilic in nature.

Figure $2 \mathrm{~b}$ shows the $\mathrm{y}-\mathrm{z}$ plane at the inlet $(x=0)$. There is a constant temperature boundary at $y=0$, and the walls to $y=L_{t}$ along the $z$-axis and to $y=L_{w}$ along the $x$-axis form a symmetric boundary condition. $z=0$ is the bottom channel surface where the patterned wettability is implemented, and a constant heat flux, $Q_{0}$, is supplied to the entire surface. There is a wall from $z=L_{h}-L_{t}$ to $z=L_{h}$, and there is a constant temperature boundary condition at $z=L_{h}$. Each geometric section can be represented by the capillary lengths: $L_{w}=0.076 L_{0}, L_{l}=0.4 L_{0}, L_{t}=0.005 L_{0}$, and $L_{h}=0.17 L_{0}$.

In Figure $2 c$, the wettability pattern is shown on the bottom channel surface $(z=0)$. The striped pattern is repeated throughout the channel. The pattern pitch is a set of hydrophobic and hydrophilic stripes of lengths $L_{p h o}$ and $L_{p h i}$, respectively. The contact angle of the hydrophobic area is $123^{\circ}$, and the contact angle of the hydrophilic area is $54^{\circ}$ [19].

The number of the grid is a $500 \times 75 \times 160$ uniform grid. The constant heat flux is $80 \mathrm{~kW} / \mathrm{m}^{2}$. The Reynolds number of the flow is 0.11 . Table 1 shows the pattern pitch and ratio of the $L_{\text {pho }}$ and $L_{\text {phi }}$ (pattern ratio) of each simulation case.

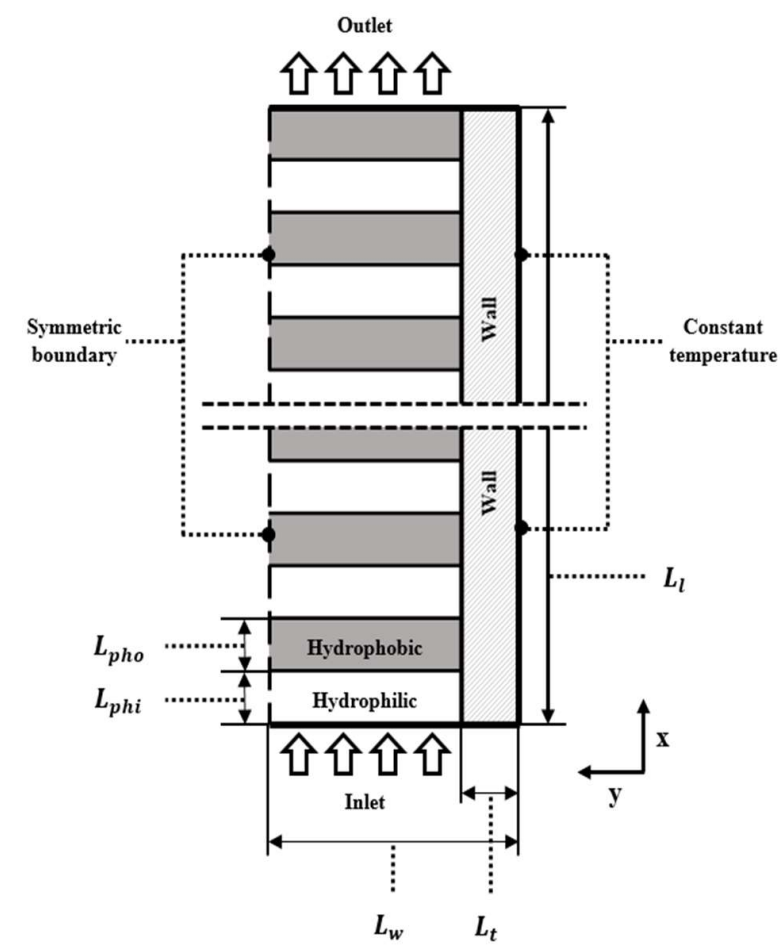

(a)

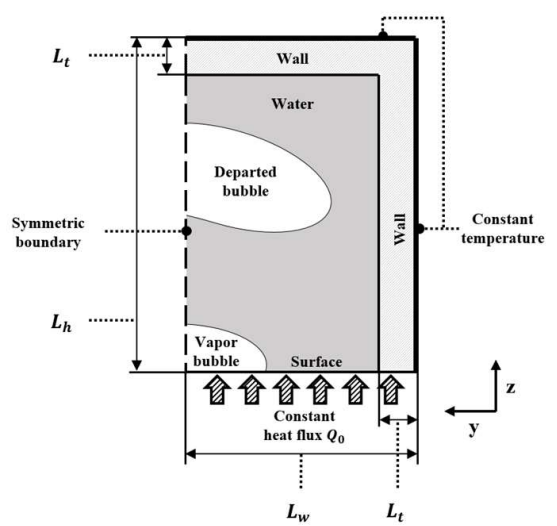

(b)

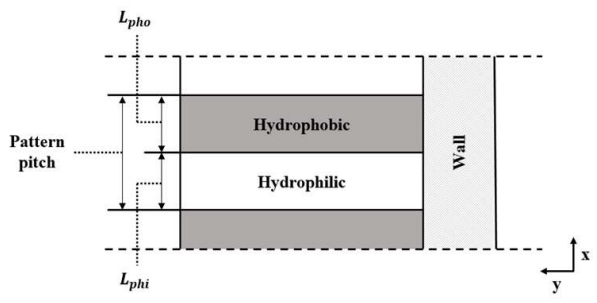

(c)

Figure 2. (a) Simulation domain description of the $x-y$ plane at $z=0$. (b) Simulation domain description of the $y$ - $z$ plane at $x=0$. (c) Pattern wettability description of the $x-y$ plane at $z=0$. 
Table 1. Patterned wettability cases.

\begin{tabular}{|c|c|c|}
\hline Case Name & Pattern Pitch $\left(L_{\text {pho }}+L_{\text {phi }}\right)$ & Pattern Ratio $\left(L_{\text {pho }} / L_{\text {phi }}\right)$ \\
\hline Case $0.01-9.00$ & \multirow{5}{*}{$0.01 L_{0}$} & $0.009 L_{0}: 0.001 L_{0}(9.00)$ \\
\hline Case $0.01-2.33$ & & $0.007 L_{0}: 0.003 L_{0}$ \\
\hline Case $0.01-1.00$ & & $0.005 L_{0}: 0.005 L_{0}(1.00)$ \\
\hline Case $0.01-0.43$ & & $0.003 L_{0}: 0.007 L_{0}(0.43)$ \\
\hline Case $0.01-0.11$ & & $0.001 L_{0}: 0.009 L_{0}(0.11)$ \\
\hline Case $0.02-9.00$ & \multirow{5}{*}{$0.02 L_{0}$} & $0.018 L_{0}: 0.002 L_{0}(9.00)$ \\
\hline Case $0.02-2.33$ & & $0.014 L_{0}: 0.006 L_{0}(2.33)$ \\
\hline Case $0.02-1.00$ & & $0.010 L_{0}: 0.010 L_{0}(1.00)$ \\
\hline Case $0.02-0.43$ & & $0.006 L_{0}: 0.014 L_{0}(0.43)$ \\
\hline Case $0.02-0.11$ & & $0.002 L_{0}: 0.018 L_{0}(0.11)$ \\
\hline Case $0.03-9.00$ & \multirow{5}{*}{$0.03 L_{0}$} & $0.027 L_{0}: 0.003 L_{0}(9.00)$ \\
\hline Case $0.03-2.33$ & & $0.021 L_{0}: 0.009 L_{0}(2.33)$ \\
\hline Case $0.03-1.00$ & & $0.015 L_{0}: 0.015 L_{0}(1.00)$ \\
\hline Case $0.03-0.43$ & & $0.009 L_{0}: 0.021 L_{0}(0.43)$ \\
\hline Case $0.03-0.11$ & & $0.003 L_{0}: 0.027 L_{0}(0.11)$ \\
\hline Case $0.04-9.00$ & \multirow{5}{*}{$0.04 L_{0}$} & $0.036 L_{0}: 0.004 L_{0}(9.00)$ \\
\hline Case $0.04-2.33$ & & $0.028 L_{0}: 0.012 L_{0}(2.33)$ \\
\hline Case $0.04-1.00$ & & $0.020 L_{0}: 0.020 L_{0}(1.00)$ \\
\hline Case $0.04-0.43$ & & $0.012 L_{0}: 0.028 L_{0}(0.43)$ \\
\hline Case $0.04-0.11$ & & $0.004 L_{0}: 0.036 L_{0}(0.11)$ \\
\hline Case $0.05-9.00$ & \multirow{5}{*}{$0.05 L_{0}$} & $0.045 L_{0}: 0.005 L_{0}(9.00)$ \\
\hline Case $0.05-2.33$ & & $0.035 L_{0}: 0.015 L_{0}(2.33)$ \\
\hline Case $0.05-1.00$ & & $0.025 L_{0}: 0.025 L_{0}(1.00)$ \\
\hline Case $0.05-0.43$ & & $0.015 L_{0}: 0.035 L_{0}(0.43)$ \\
\hline Case $0.05-0.11$ & & $0.005 L_{0}: 0.045 L_{0}(0.11)$ \\
\hline Case 0.07-9.00 & \multirow{5}{*}{$0.07 L_{0}$} & $0.063 L_{0}: 0.007 L_{0}(9.00)$ \\
\hline Case $0.07-2.33$ & & $0.049 L_{0}: 0.021 L_{0}(2.33)$ \\
\hline Case $0.07-1.00$ & & $0.035 L_{0}: 0.035 L_{0}(1.00)$ \\
\hline Case $0.07-0.43$ & & $0.021 L_{0}: 0.049 L_{0}(0.43)$ \\
\hline Case $0.07-0.11$ & & $0.007 L_{0}: 0.063 L_{0}(0.11)$ \\
\hline Case $0.1-9.00$ & \multirow{5}{*}{$0.1 L_{0}$} & $0.09 L_{0}: 0.01 L_{0}(9.00)$ \\
\hline Case $0.1-2.33$ & & $0.07 L_{0}: 0.03 L_{0}(2.33)$ \\
\hline Case $0.1-1.00$ & & $0.05 L_{0}: 0.05 L_{0}(1.00)$ \\
\hline Case $0.1-0.43$ & & $0.03 L_{0}: 0.07 L_{0}(0.43)$ \\
\hline Case $0.1-0.11$ & & $0.01 L_{0}: 0.09 L_{0}(0.11)$ \\
\hline
\end{tabular}

Note: Case name-The capillary length ratio of the pattern pitch and the pattern ratio in the pattern pitch.

\section{Results}

\subsection{Validation}

The results for bubble nucleation and bubble nucleation heat transfer were compared with experimental results to validate the numerical model. First, the analytical equation for the bubble departure diameter from the study of Hazi et al. [28] was compared with the simulation results. The simulation was performed in a single hydrophobic dot domain. The z-direction length of the domain was $L_{\mathrm{h}}=8.51 L_{0}$, the width of the hydrophobic dot was $0.28 L_{0}$, and the $x$ - and $y$-direction lengths of the domain were both $L_{\mathrm{W}}=11.26 L_{0}$. The constant heat flux boundary had a width of $L_{\mathrm{f}}$ $=4.54 L_{0}$ [20]. Table 2 lists the physical properties, density ratio of vapor and liquid, surface tension, viscosity, and thermal diffusivity used in the simulations.

Relaxation times are obtained as follows: 


$$
\begin{gathered}
v=c_{S}^{2}(\tau-0.5) \delta_{t} \\
\alpha=c_{s}^{2}\left(\tau_{T}-0.5\right) \delta_{t}
\end{gathered}
$$

where $v$ is kinematic viscosity and $\alpha$ is the thermal diffusivity.

Table 2. Physical properties of the conducted simulations.

\begin{tabular}{cc}
\hline Properties & Values \\
\hline Thermal diffusivity of solid & $4.05 \times 10^{-6} \mathrm{~m}^{2} / \mathrm{s}$ \\
Thermal diffusivity of liquid & $1.47 \times 10^{-7} \mathrm{~m}^{2} / \mathrm{s}$ \\
Thermal diffusivity of vapor & $2.05 \times 10^{-5} \mathrm{~m}^{2} / \mathrm{s}$ \\
Kinetic viscosity of liquid & $8.58 \times 10^{-7} \mathrm{~m}^{2} / \mathrm{s}$ \\
Kinetic viscosity of vapor & $2.02 \times 10^{-5} \mathrm{~m}^{2} / \mathrm{s}$ \\
Surface tension & $5.9 \times 10^{-5} \mathrm{~m}^{2} / \mathrm{s}$ \\
Density ratio: liquid/vapor & 1300 \\
\hline
\end{tabular}

Figure 3 shows the bubble departure diameter with a gravitational force $g$. This result was consistent with the results from a previous study [29]. The bubble departure diameter $D_{\mathrm{d}}$ approximated by $g^{-0.5}$, was consistent with the theoretical equation given in Equation (23).

$$
D_{\mathrm{d}}=\sqrt{\frac{\gamma}{g\left(\rho_{1}-\rho_{g}\right)}}
$$

In Figure 4, the boiling curve from the experimental study of Jo et al. [19] was compared with the results in this study. The boiling curve is divided by the convection regime and the nucleate boiling regime. Heat transfer occurred by only natural convection in the convection regime, i.e., at lower heat fluxes without bubbles [30,31]. In the nucleate boiling regime, forced convection occurred through bubble nucleation. The Figure 4 shows that the numerical data is consistent with the experimental results [19].

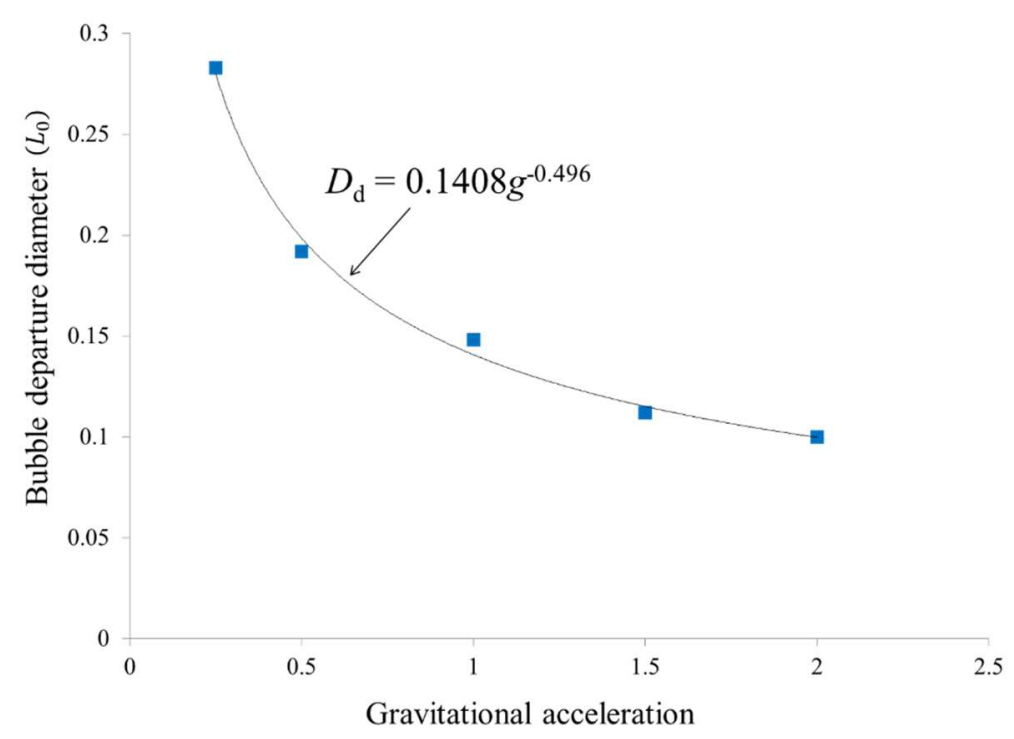

Figure 3. Departure diameter as a function of gravitational acceleration. Reprinted with permission from [20]. Copyright 2018 Elsevier. 


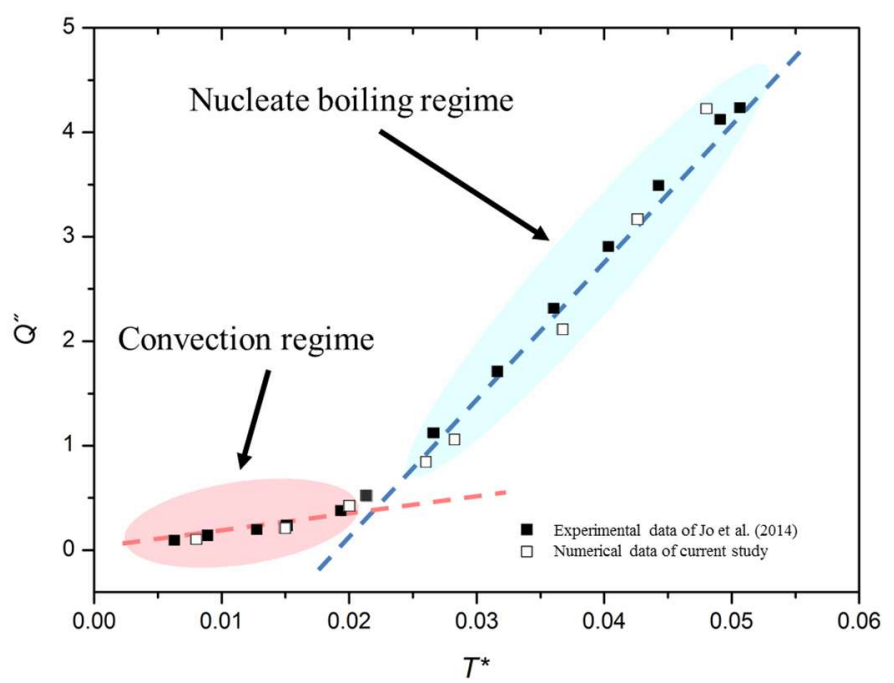

Figure 4. Comparison of the experimental data from Jo et al. and the numerical data of the current study. Reprinted with permission from [19]. Copyright 2018 Elsevier. The convection regime and the nucleate boiling regime are indicated by shading, and the slopes of both regimes are indicated by dashed lines. Reprinted with permission from [20]. Copyright 2018 Elsevier.

\subsection{Effect of Patterned Wettability}

\subsubsection{Pattern Ratio}

Because surface wettability is an important factor for bubble nucleation, controlling the wettability pattern strongly affects the phase change phenomena. In this section, the effect of the pattern ratio, which is a size ratio of hydrophobic area and hydrophilic area, is analyzed. Figure 5 shows types of bubble nucleation on patterned surfaces with different pattern ratios. These cases were compared with an equivalent pattern pitch of 0.03 and a time step of 30,000. Bright green, bright blue, and dark blue correspond to the hydrophobic surface, hydrophilic surface, and channel wall, respectively. At this stage, the bubbles were nucleated on the hydrophobic surface. The nucleated bubbles become smaller as the pattern ratio was lowered. Thus, it was confirmed that the size of the hydrophobic area influenced the size of the nucleated bubble. Furthermore, as size of the hydrophobic area got smaller, the bubble nucleation time was delayed.

Figure 6 shows the boiling curve with superheat and heat flux. In this simulation, the phase change was cyclic and was stabilized in the superheat and heat flux of the narrow section. Therefore, the types of phase change could be compared by looking at the maximum superheat and heat flux. The region at which the superheat decreased is where the maximum size bubble and the highest heat transfer coefficient were observed. These results show that the lower maximum superheat was observed in Case 0.03-9.00, so a relatively high heat transfer coefficient could be expected.

Figure 7 shows the normalized heat transfer coefficient as a function of time and confirms the periodicity of bubble nucleation. The heat transfer coefficient increased when the bubble was generated and decreased when the bubble departed. This periodicity is identically observed in all cases. However, as the pattern ratio decreased, the heat transfer coefficient trend also decreased. Examining the averaged heat transfer coefficient, the highest value in Case 0.03-9.00 and the lowest value in Case $0.03-0.11$ were observed. The averaged heat transfer coefficient gradually decreased to a pattern ratio of 1.00 (Case $0.03-1.00$ ) and sharply decreased after the pattern ratio of 1.00 (Case $0.03-1.00)$. This was due to bubble nucleation delay. 


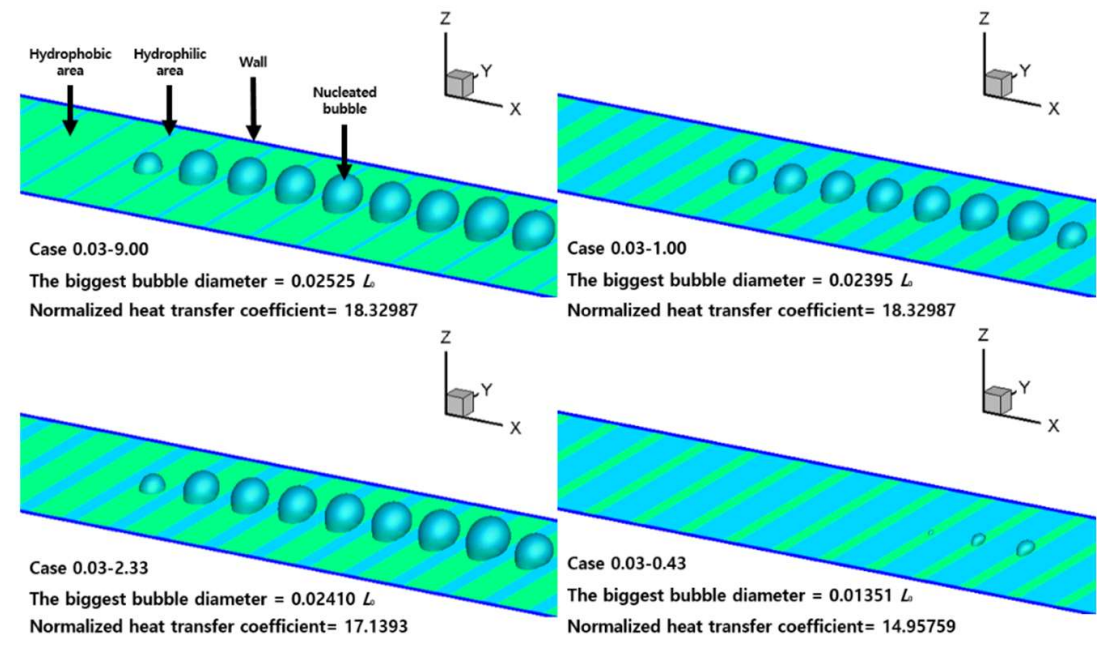

Figure 5. Comparison of bubble nucleation type and biggest bubble diameter according to pattern ratio. Time step $=30,000$ and the pattern pitch is $0.03 L_{0}$.

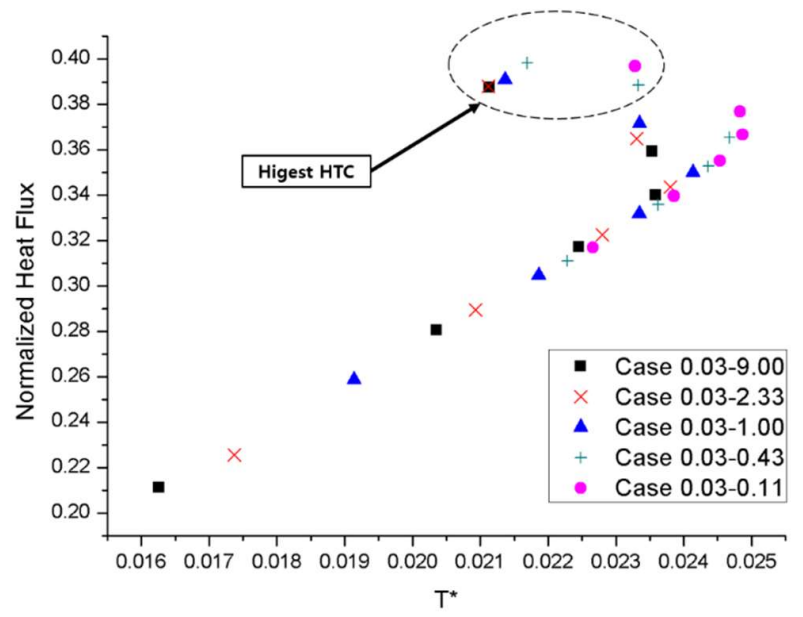

Figure 6. Boiling curves with superheat and heat flux graph according to the pattern ratio in $0.03 L_{0}$ pattern pitch.

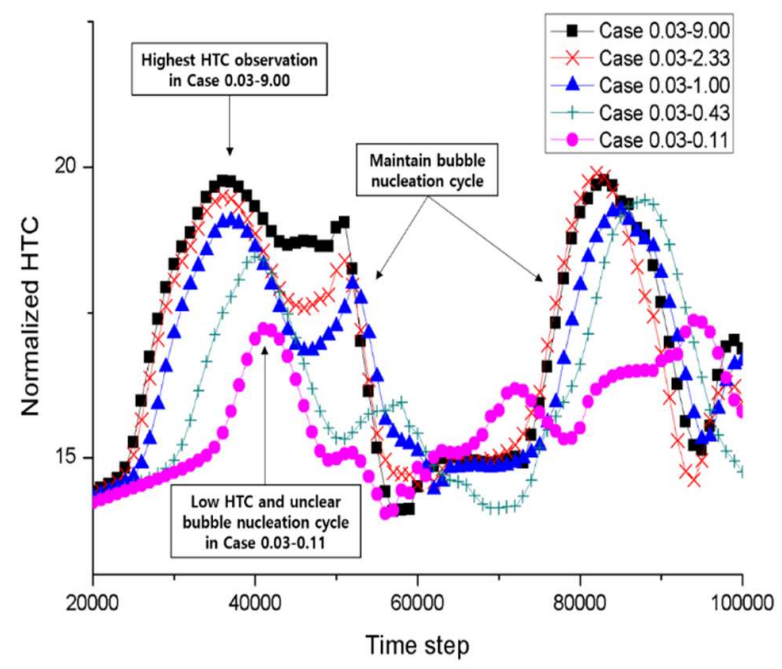

Figure 7. Normalized heat transfer coefficient vs. time step graph. 


\subsubsection{Pattern Pitch}

Figure 8 shows the variation of the boiling curve depending on the pattern pitch. The pattern ratio was identical to the pattern ratio of 9.00, which showed the highest heat transfer in Section 4.2.1. As the pattern pitch increased, the hydrophilic area increased, and the maximum super heat decreased. However, the change of the boiling curve according to the pattern pitch was relatively small because the ratio of the hydrophobic area was high due to the high pattern ratio.

Figure 9 indicates the average Normalized Heat Transfer Coefficient (NHTC) of each pattern pitch. From pitch 0.01 to 0.03 , the average NHTC sharply increased, and then from pitch 0.03 , the average NHTC gradually decreased. At pitch 0.03 (Case 0.03-9.00), the highest average NHTC was observed.

The bubble nucleation and the surface temperature with pattern pitch are shown in Figure 10 with pitch 0.01 (Case 0.01-9.00), pitch 0.03 (Case 0.03-9.00), and pitch (Case 0.05-9.00) at a time step of 30,000 . Bubbles individually nucleated on the hydrophobic area in pitch 0.03 and pitch 0.05 , whereas in pitch 0.01 , bubbles coalesced and then slid as soon as they nucleated. As bubbles absorbed more heat from their surroundings, they grew bigger. Therefore, in pitch 0.05 , the bubble size was larger and the surface temperature below the bubble was lower. However, in pitch 0.03 , the averaged surface temperature had the lowest value due to the number of bubble nucleation.

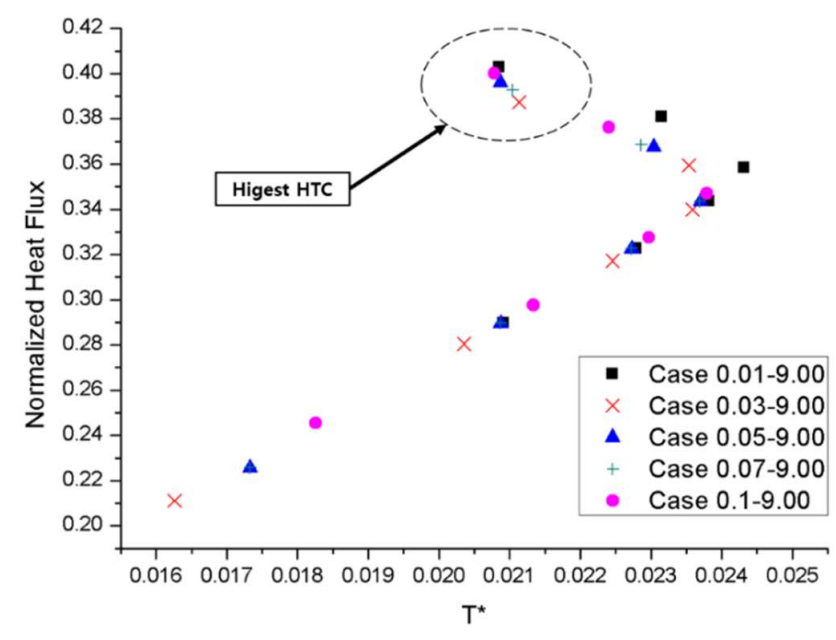

Figure 8. Boiling curves on a heat flux vs. superheat graph according to the pattern pitch 9.00 pattern ratio.

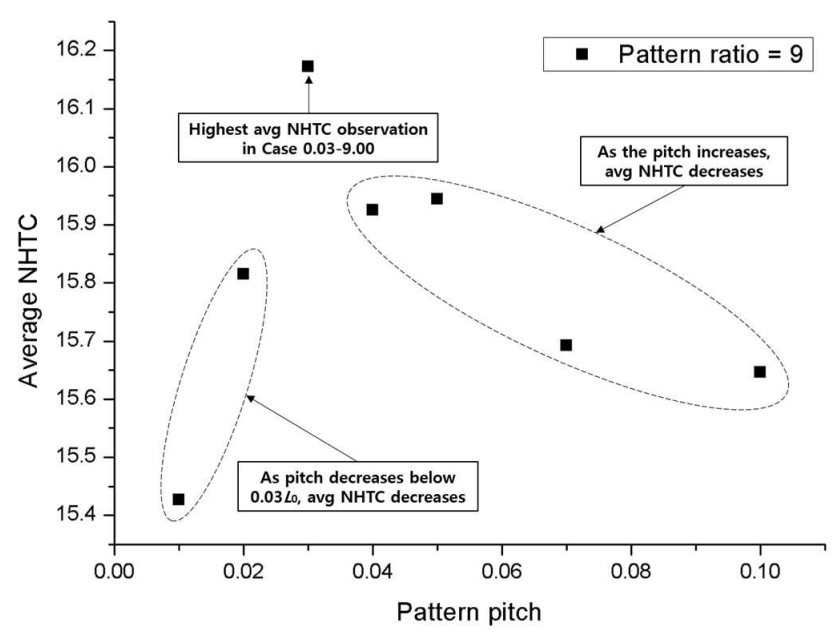

Figure 9. Average normalized heat transfer coefficient according to the pattern pitch in 9.00 pattern ratio. 


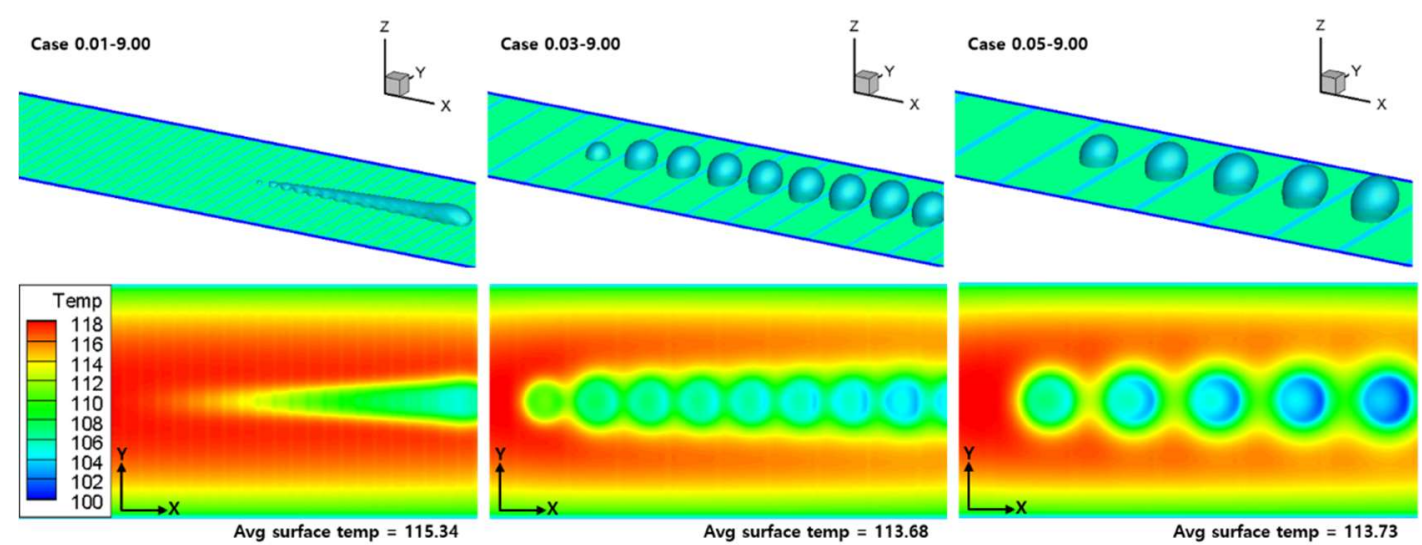

Figure 10. Bubble nucleation and surface temperature according to pattern pitch; Time step $=30,000$; 9.00 pattern ratio.

\section{Discussion}

Figure 5 shows that the hydrophobic area had a significant effect on bubble nucleation. At the same pattern pitch, the bubble diameter increased as the pattern ratio increased. In Case 0.03-0.43 and Case 0.03-0.11, bubble nucleation was not observed at the same time step. These characteristics are also shown in the boiling curve of Figure 6. In Case 0.03-2.33 and Case 0.03-1.00, in which bubble nucleation occurred normally, a boiling curve similar to Case $0.03-9.00$ was observed. In Figure 7, the NHTC changes with the pattern ratio. As with the previous results, the average NHTC increased as the pattern ratio increased, and decreased sharply as the pattern ratio decreased. In Case 0.03-9.00, the average NHTC was higher than that of other cases. This is due to the bubble nucleation-departure cycle in Figure 7. In the cases of patterned wettability, there is a difference in the NHTC but the cycle is maintained. In Case 0.03-0.11, it can be seen that the cycle was not maintained because the heat transfer was adversely affected by the occurrence of uneven bubbles after the first nucleation.

Figure 8 shows the boiling curve with the pattern pitch as well as a similar trend in all cases. However, the increase in pattern ratio also increased the hydrophobic area, and the boiling curve shows a similar pattern.

Figure 9 plots the average heat transfer coefficients with the pattern pitch. The pattern ratio was fixed at 9.00. The highest average heat transfer coefficient was observed in Case 0.03-9.00, and the average heat transfer coefficient decreased at higher and lower pattern pitches. The cause of this result can be found in Figure 10, which shows bubble nucleation and surface temperature. As shown in Figure 10, the larger the hydrophobic area, the larger the diameter of the bubble. In other words, the amount of generated bubbles increased as the pattern pitch at the same channel length decreased. The increase of the contact area, due to the generation of many bubbles, has a positive effect on the heat transfer of the surface. This can be confirmed by the lower surface temperature. If the pattern pitch decreases below a certain level, the hydrophobic area decreases. This means that the distance between the nucleated bubbles is close enough to quickly coalescence. This reduces the heat transfer efficiency.

\section{Conclusions}

In this study, the optimization of heat transfer through a hydrophobic pattern was performed on a micro channel surface with hydrophilic characteristics. The pattern cases were divided into a pattern pitch, representing the overall length of the pattern, and a pattern ratio, representing the ratio of hydrophobic to hydrophilic. Pattern optimization requires several conditions.

- From the viewpoint of heat transfer, the wider the hydrophobic area, the better. Therefore, an excellent heat transfer coefficient is observed on a hydrophobic surface with a high pattern 
ratio. At certain pattern ratios, the heat transfer coefficient is better than the uniform hydrophobic surface because the bubble nucleation-departure cycle is stable due to the pattern ratio effect.

- The amount of generated bubbles increases as the pattern pitch decreases. This is effective for surface heat transfer. However, when the pattern pitch is reduced below $0.03 L_{0}$, the amount of bubbles decreases due to an insufficient hydrophobic area to generate bubbles. As bubble generation decreases, surface heat transfer also decreases. From these results, it was concluded that finding an optimal area is necessary for determining the pattern pitch.

- To optimize channel heat transfer, patterned wettability must be applied to widen the hydrophobic area while keeping the bubble nucleation-bubble departure cycle uniform. It is also necessary to use the minimum pattern pitch where many bubbles are generated. However, a pattern pitch of less than a certain size reduces the effect of the hydrophobic area and hinders the generation of individual bubbles, which adversely affects heat transfer.

The optimization in this study is based on a simple stripe pattern. Stripes oriented perpendicular to the flow were able to bring about pattern optimization by affecting the bubble nucleation-departure cycle. Potential future studies include investigating the application and optimization of different types of patterns depending on the shape of the channel or the trend of the flow.

Author Contributions: Conceptualization, Y.J.W.; Methodology, J.S.L.; Software, Y.J.W. and J.S.L.; Validation, J.S.L.; Formal Analysis, Y.J.W.; Investigation, Y.J.W. and J.H.K.; Resources, J.S.L.; Data Curation, Y.J.W., J.H.K. and J.S.L.; Writing-Original Draft Preparation, Y.J.W. and J.H.K.; Writing-Review \& Editing, Y.J.W., J.H.K. and J.S.L.; Visualization, Y.J.W.; Supervision, J.S.L.; Project administration, J.S.L.

Funding: This work was funded by the Advanced Research Center Program (NRF-2015R1A5A1037668) through a National Research Foundation of Korea (NRF) grant funded by the Ministry of Science, ICT, and Future Planning (MSIP).

Conflicts of Interest: The authors declare no conflict of interest.

\section{Appendix A}

\section{First Order Distribution Function [24]}

Statistical Mechanics offers a statistical approach in which we represent a system by an ensemble of many copies. The distribution $f^{(1)}(x, p, t)$ gives the probability of finding a particular molecule with a given position and momentum; the positions and momenta of the remaining $N-1$ molecules can remain unspecified because no experiment can distinguish between molecules, so the choice of which molecule does not matter. This is the 'Single particle' distribution function. $f^{(1)}$ is adequate for describing all gas properties that do not depend on relative positions of molecules (dilute gas with long mean free path).

The probable number of molecules with position coordinates in the range $x \pm d x$ and momentum coordinates $p \pm d p$ is given by $f^{(1)}(x, p, t) d x d p$. Say we introduce an external force $F$ that is small relative to intermolecular forces. If there are no collisions, then at time $t+d t$, the new positions of molecules starting at $x$ are $x+(p / m) d t=x+(d x / d t) d t=x+d x$ and the new momenta are $p=p+F d t=p$ $+(d p / d t) d t=p+d p$.

Thus, when the positions and momenta are known at a particular time $t$, incrementing them allows us to determine $f^{(1)}$ at a future time $t+d t$ :

$$
f^{(1)}(x+d x, p+d p, t+d t) d x d p=f^{(1)}(x, p, t) d x d p
$$

This is the streaming process.

There are however collisions that result in some phase points starting at $(x, p)$ not arriving at $(x+p / m d t, p+F d t)=(x+d x, p+d p)$ and some not starting at $(x, p)$ arriving there too. We set $\Gamma^{(-)} d x d p d t$ equal to the number of molecules that do not arrive in the expected portion of phase space due to collisions during time $d t$. Similarly, we set $\Gamma^{(+)} d x d p d t$ equal to the number of molecules that 
start somewhere other than $(x, p)$ and arrive in that portion of phase space due to collisions during time $d t$. If we start with Equation (A1) and add the changes in $f^{(1)}$ due to these collisions we obtain:

$$
\begin{gathered}
f^{(1)}(x+d x, p+d p, t+d t) d x d p \\
=f^{(1)}(x, p, t) d x d p+\left[\Gamma^{(+)}-\Gamma^{(-)}\right] d x d p d t
\end{gathered}
$$

The first order terms of a Taylor series expansion of the LHS (Left-hand side) of Equation (A2):

$$
\begin{gathered}
f^{(1)}(x+d x, p+d p, t+d t) \\
=f^{(1)}(x, p, t)+d x \cdot \nabla_{x} f^{(1)}+d p \cdot \nabla_{p} f^{(1)}+\left(\frac{\partial f^{(1)}}{\partial t}\right) d t+\cdots
\end{gathered}
$$

Give the Boltzmann equation:

$$
\begin{gathered}
{\left[f^{(1)}(x, p, t)+d x \cdot \nabla_{x} f^{(1)}+d p \cdot \nabla_{p} f^{(1)}+\left(\frac{\partial f^{(1)}}{\partial t}\right) d t+\cdots\right] d x d p} \\
=f^{(1)}(x, p, t) d x \mathrm{~d} p+\left[\Gamma^{(+)}-\Gamma^{(-)}\right] d x \mathrm{~d} p d t
\end{gathered}
$$

or,

$$
v \cdot \nabla_{x} f^{(1)}+F \cdot \nabla_{p} f^{(1)}+\frac{\partial f^{(1)}}{\partial t}=\Gamma^{(+)}-\Gamma^{(-)}
$$

Note that this can be derived for an arbitrary number of different chemical components as well.

In its complete form with the collision operator written more explicitly, the Boltzmann equation is a nonlinear integral differential equation and is particularly complicated. According to Harris [32], 50 years elapsed from the time that Boltzmann derived the equation before an approximate solution was found. With lattice Boltzmann methods, we approximately solve the equation from the particle perspective and focus on an equation strongly akin to Equation (A2); it explicitly contains the 'collide and stream' notion central to LBM (Lattice Boltzmann method).

\section{References}

1. Sung, M.K.; Mudawar, I. Single-phase and two-phase hybrid cooling schemes for high-heat-flux thermal management of defense electronics. J. Electron. Packag. 2009, 131, 021013. [CrossRef]

2. Fuchs, T.; Kern, J.; Stephan, P. A transient nucleate boiling model including microscale effects and wall heat transfer. J. Heat Transf. 2006, 128, 1257-1265. [CrossRef]

3. Kandlikar, S.G. A scale analysis based theoretical force balance model for critical heat flux (CHF) during saturated flow boiling in micro channels and minichannels. J. Heat Transf. 2010, 132, 081501. [CrossRef]

4. Kim, S.M.; Mudawar, I. Review of databases and predictive methods for heat transfer in condensing and boiling mini/micro-channel flows. Int. J. Heat Mass Transf. 2014, 77, 627-652. [CrossRef]

5. Deng, D.; Wan, W.; Qin, Y.; Zhang, J.; Chu, X. Flow boiling enhancement of structured microchannels with micro pin fins. Int. J. Heat Mass Transf. 2017, 105, 338-349. [CrossRef]

6. Xu, F.; $\mathrm{Wu}, \mathrm{H}$.; Liu, Z. Flow patterns during flow boiling instability in silicon-based pin-fin microchannels. J. Heat Transf. 2018, 140, 031501. [CrossRef]

7. Lin, P.; Fu, B.; Pan, C. Critical heat flux on flow boiling of methanol-water mixtures in diverging microchannel with artificial cavities. Int. J. Heat Mass Transf. 2011, 54, 3156-3166. [CrossRef]

8. Deng, D.; Tang, Y.; Liang, D.; He, H.; Yang, S. Flow boiling characteristics in porous heat sink with reentrant microchannels. Int. J. Heat Mass Transf. 2014, 70, 463-477. [CrossRef]

9. Kim, D.E.; Yu, D.I.; Jerng, D.W.; Kim, M.H.; Ahn, H.S. Review of boiling heat transfer enhancement on micro/nanostructured surfaces. Exp. Therm. Fluid Sci. 2015, 66, 173-196. [CrossRef]

10. Zhou, K.; Coyle, C.; Li, J.; Buongiorno, J.; Li, W. Flow boiling in vertical narrow microchannels of different surface wettability characteristics. Int. J. Heat Mass Transf. 2017, 109, 102-114. [CrossRef]

11. Park, H.M.; Jeong, Y.H. Flow boiling CHF enhancement by wettability and flow conditions in a slug flow in the rectangular curved channel. Exp. Therm. Fluid Sci. 2018, 91, 388-398. [CrossRef] 
12. Li, Q.; Kang, Q.; Francois, M.M.; He, Y.; Luo, K. Lattice Boltzmann modeling of boiling heat transfer: The boiling curve and the effects of wettability. Int. J. Heat Mass Transf. 2015, 85, 787-796. [CrossRef]

13. Gong, S.; Cheng, P. Lattice Boltzmann simulations for surface wettability effects in saturated pool boiling heat transfer. Int. J. Heat Mass Transf. 2015, 85, 635-646. [CrossRef]

14. Takata, Y.; Hidaka, S.; Masuda, M.; Ito, T. Pool boiling on a superhydrophilic surface. Int. J. Energy Res. 2003, 27, 111-119. [CrossRef]

15. Phan, H.T.; Caney, N.; Marty, P.; Colasson, S.; Gavillet, J. Wettability control by nanocoating: The effects on pool boiling heat transfer and nucleation mechanism. Int. J. Heat Mass Transf. 2009, 52, 5459-5471. [CrossRef]

16. Vazquez-Martinez, J.M.; Salguero Gomez, J.; Mayuet Ares, P.F.; Fernandez-Vidal, S.R.; Batista Ponce, M. Effects of laser microtexturing on the wetting behavior of Ti6Al4V alloy. Coatings 2018, 8, 145. [CrossRef]

17. Li, J.; Yu, W.; Zheng, D.; Zhao, X.; Choi, C.; Sun, G. Hot embossing for whole Teflon superhydrophobic surfaces. Coatings 2018, 8, 227. [CrossRef]

18. Nam, Y.; Ju, Y.S. Bubble nucleation on hydrophobic islands provides evidence to anomalously high contact angles of nanobubbles. Appl. Phys. Lett. 2008, 93, 103115. [CrossRef]

19. Jo, H.; Kim, S.; Park, H.S.; Kim, M.H. Critical heat flux and nucleate boiling on several heterogeneous wetting surfaces: Controlled hydrophobic patterns on a hydrophilic substrate. Int. J. Multiph. Flow 2014, 62, 101-109. [CrossRef]

20. Lee, J.S.; Lee, J.S. Numerical approach to the suppression of film boiling on hot-spots by radial control of patterned wettability. Int. J. Multiph. Flow 2016, 84, 165-175. [CrossRef]

21. Gong, S.; Cheng, P. A lattice Boltzmann method for simulation of liquid-vapor phase-change heat transfer. Int. J. Heat Mass Transf. 2012, 55, 4923-4927. [CrossRef]

22. Li, Q.; Luo, K.H.; Li, X.J. Lattice Boltzmann modeling of multiphase flows at large density ratio with an improved pseudopotential model. Phys. Rev. E 2013, 87, 053301. [CrossRef] [PubMed]

23. Xu, A.; Zhao, T.S.; An, L.; Shi, L. A three-dimensional pseudo-potential-based lattice Boltzmann model for multiphase flows with large density ratio and variable surface tension. Int. J. Heat Fluid Flow 2015, 56, 261-271. [CrossRef]

24. Sukop, M.C.; Thorne, D.T., Jr. Lattice Boltzmann Modeling: An Introduction for Geoscientists and Engineers, 2nd ed.; Springer: Heidelberg, Germany, 2006.

25. Kupershtokh, A.L.; Medvedev, D.A. Lattice Boltzmann equation method in electrohydrodynamic problems. J. Electrost. 2006, 64, 581-585. [CrossRef]

26. Gong, S.; Cheng, P. Numerical investigation of droplet motion and coalescence by an improved lattice Boltzmann model for phase transitions and multiphase flows. Comput. Fluids 2012, 53, 93-104. [CrossRef]

27. Lee, J.S.; Moon, J.Y.; Lee, J.S. Study of transporting of droplets on heterogeneous surface structure using the lattice Boltzmann approach. Appl. Therm. Eng. 2014, 72, 104-113. [CrossRef]

28. Hazi, G.; Markus, A. On the bubble departure diameter and release frequency based on numerical simulation results. Int. J. Heat Mass Transf. 2009, 52, 1472-1480. [CrossRef]

29. Di Bari, S.; Robinson, A.J. Robinson, Experimental study of gas injected bubble growth from submerged orifices. Exp. Therm. Fluid Sci. 2013, 44, 124-137. [CrossRef]

30. Bourdon, B.; Rioboo, R.; Marengo, M.; Gosselin, E.; De Coninck, J. Influence of the wettability on the boiling onset. Langmuir 2012, 28, 1618-1624. [CrossRef] [PubMed]

31. Bourdon, B.; Di Marco, P.; Riobóo, R.; Marengo, M.; De Coninck, J. Enhancing the onset of pool boiling by wettability modification on nanometrically smooth surfaces. Int. Commun. Heat Mass Transf. 2013, 45, 11-15. [CrossRef]

32. Harris, S. An Introduction to the Theory of the Boltzmann Equation; Courier Corporation: North Chelmsford, MA, USA, 2004.

(C) 2018 by the authors. Licensee MDPI, Basel, Switzerland. This article is an open access article distributed under the terms and conditions of the Creative Commons Attribution (CC BY) license (http://creativecommons.org/licenses/by/4.0/). 